This item is the archived peer-reviewed author-version of:

\title{
Peripheral blood collection : the first step towards gene expression profiling
}

\section{Reference:}

Franken Carmen, Remy Sylvie, Lambrechts Nathalie, Hollanders Karen, Den Hond Elly, Schoeters Greet.- Peripheral blood collection : the first step towards gene expression profiling

Biomarkers: biochemical indicators of exposure, response, and susceptibility to chemicals - ISSN 1354-750X - (2016), p. 1-8 Full text (Publishers DOI): http://dx.doi.org/doi:10.3109/1354750X.2016.1153721 


\section{PROOF COVER SHEET}

Author(s): Carmen Franken, Sylvie Remy, Nathalie Lambrechts, Karen Hollanders, Elly Den Hond, and Greet Schoeters

Article title: Peripheral blood collection: the first step towards gene expression profiling

Article no: IBMK_A_1153721

Enclosures: 1) Query sheet

2) Article proofs

Dear Author,

1. Please check these proofs carefully. It is the responsibility of the corresponding author to check these and approve or amend them. A second proof is not normally provided. Taylor \& Francis cannot be held responsible for uncorrected errors, even if introduced during the production process. Once your corrections have been added to the article, it will be considered ready for publication.

Please limit changes at this stage to the correction of errors. You should not make trivial changes, improve prose style, add new material, or delete existing material at this stage. You may be charged if your corrections are excessive (we would not expect corrections to exceed 30 changes).

For detailed guidance on how to check your proofs, please paste this address into a new browser window: http://journalauthors.tandf.co.uk/production/checkingproofs.asp

Your PDF proof file has been enabled so that you can comment on the proof directly using Adobe Acrobat. If you wish to do this, please save the file to your hard disk first. For further information on marking corrections using Acrobat, please paste this address into a new browser window: http://journalauthors.tandf.co.uk/production/ acrobat.asp

2. Please review the table of contributors below and confirm that the first and last names are structured correctly and that the authors are listed in the correct order of contribution. This check is to ensure that your name will appear correctly online and when the article is indexed.

\begin{tabular}{|c|c|c|c|c|}
\hline Sequence & Prefix & Given name(s) & Surname & Suffix \\
\hline 1 & & Carmen & Franken & \\
\hline 2 & & Sylvie & Remy & \\
\hline 3 & & Nathalie & Lambrechts & \\
\hline 4 & & Karen & Hollanders & \\
\hline 5 & & Elly & Den Hond & \\
\hline 6 & & Greet & Schoeters & \\
\hline
\end{tabular}

Queries are marked in the margins of the proofs, and you can also click the hyperlinks below.

\section{General points:}

1. Permissions: You have warranted that you have secured the necessary written permission from the appropriate copyright owner for the reproduction of any text, illustration, or other material in your article. Please see http:// journalauthors.tandf.co.uk/permissions/usingThirdPartyMaterial.asp. 
2. Third-party content: If there is third-party content in your article, please check that the rightsholder details for re-use are shown correctly.

3. Affiliation: The corresponding author is responsible for ensuring that address and email details are correct for all the co-authors. Affiliations given in the article should be the affiliation at the time the research was conducted. Please see http://journalauthors.tandf.co.uk/preparation/writing.asp.

4. Funding: Was your research for this article funded by a funding agency? If so, please insert 'This work was supported by <insert the name of the funding agency in full >', followed by the grant number in square brackets '[grant number xxxx]'.

5. Supplemental data and underlying research materials: Do you wish to include the location of the underlying research materials (e.g. data, samples or models) for your article? If so, please insert this sentence before the reference section: 'The underlying research materials for this article can be accessed at $<$ full link $>/$ description of location [author to complete]'. If your article includes supplemental data, the link will also be provided in this paragraph. See < http://journalauthors.tandf.co.uk/preparation/multimedia.asp $>$ for further explanation of supplemental data and underlying research materials.

6. The PubMed (http://www.ncbi.nlm.nih.gov/pubmed) and CrossRef databases (www.crossref.org/) have been used to validate the references. Changes resulting from mismatches are tracked in red font.

\section{AUTHOR QUERIES}

Q1: $\quad$ Please specify the author responsible for statistical design and analysis.

Q2: $\quad$ Please provide the town and state abbreviation (for US) or town and country of origin (for other countries) identifying the headquarter location for "Invitrogen, Agilent 2100 Expert Software, Agilent Technologies, NanoDrop spectrophotometer, Agilent Feature Extraction Software (Version 10.7), Matlab, Applied Biosystems, Roche LightCycler480, LightCycler software".

Q3: $\quad$ Please check the publisher location for ref. "Burczynski and Rockett (2006)".

Q4: Please check the page range for refs. "Opitz et al. (2010), Vartanian et al. (2009)".

Q5: Artwork will only appear in colour in the online journal, please ensure the description of Figure [2] will make sense when it is printed in black and white, amending captions and text as necessary.

\section{How to make corrections to your proofs using Adobe Acrobat/Reader}

Taylor \& Francis offers you a choice of options to help you make corrections to your proofs. Your PDF proof file has been enabled so that you can mark up the proof directly using Adobe Acrobat/Reader. This is the simplest and best way for you to ensure that your corrections will be incorporated. If you wish to do this, please follow these instructions:

1. Save the file to your hard disk.

2. Check which version of Adobe Acrobat/Reader you have on your computer. You can do this by clicking on the "Help" tab, and then "About".

If Adobe Reader is not installed, you can get the latest version free from http://get.adobe.com/reader/.

3. If you have Adobe Acrobat/Reader 10 or a later version, click on the "Comment" link at the right-hand side to view the Comments pane. 
4. You can then select any text and mark it up for deletion or replacement, or insert new text as needed. Please note that these will clearly be displayed in the Comments pane and secondary annotation is not needed to draw attention to your corrections. If you need to include new sections of text, it is also possible to add a comment to the proofs. To do this, use the Sticky Note tool in the task bar. Please also see our FAQs here: http://journalauthors.tandf.co.uk/production/index.asp.

5. Make sure that you save the file when you close the document before uploading it to CATS using the "Upload File" button on the online correction form. If you have more than one file, please zip them together and then upload the zip file. If you prefer, you can make your corrections using the CATS online correction form.

\section{Troubleshooting}

Acrobat help: http://helpx.adobe.com/acrobat.html

Reader help: http://helpx.adobe.com/reader.html

Please note that full user guides for earlier versions of these programs are available from the Adobe Help pages by clicking on the link "Previous versions" under the "Help and tutorials" heading from the relevant link above. Commenting functionality is available from Adobe Reader 8.0 onwards and from Adobe Acrobat 7.0 onwards.

Firefox users: Firefox's inbuilt PDF Viewer is set to the default; please see the following for instructions on how to use this and download the PDF to your hard drive: http://support.mozilla.org/en-US/kb/view-pdf-files-firefox-without-downloading-them\#w_using-a-pdf-reader-plugin 


\section{Peripheral blood collection: the first step towards gene expression profiling}

a Unit Environmental Risk and Health, Flemish Institute for Technological Research (VITO NV), Mol, Belgium; ${ }^{b}$ Department of Biomedical Sciences; ' Department of Epidemiology and Social Medicine, University of Antwerp, Antwerp, Belgium; ${ }^{d}$ Department of Environmental Medicine, University of Southern Denmark, Odense, Denmark

ARTICLE HISTORY
Received 17 August 2015
Revised 15 January 2016
Accepted 29 January 2016
Published online
KEYwORDS
Bench time; biomarker;
human blood sampling; IL-8;
RNA stability

\section{ABSTRACT}

A crucial challenge for gene expression analysis in human biomonitoring studies on whole blood samples is rapid sample handling and mRNA stabilization. This study was designed to evaluate the impact of short bench times (less than $30 \mathrm{~min}$ ) on yield, quality and gene expression of mRNA in the presence of different stabilization buffers (Tempus ${ }^{T M}$ Blood RNA tube and RNAlater ${ }^{\circledR}$ Stabilization Reagent). Microarray analyzes showed significant changes over short periods of time in expression of a considerate part of the transcriptome (2356 genes) with a prominent role for NFKB-, cancer- and glucocorticoidmediated networks, and specifically interleukin-8 (IL-8). These findings suggest that even short bench times affect gene expression, requiring to carry out blood collection in a strictly standardized way.
ARTICLE HISTORY

Received 17 August 2015

Published online 【 I

Bench time; biomarker; RNA stability

\section{Introduction}

Transcriptional profiling of whole blood has emerged as a powerful tool for biomarker discovery in clinical medicine and health research (Bai et al., 2013; Ellinger-Ziegelbauer et al., 2009; McHale et al., 2010; Sullivan et al., 2006; Tang et al., 2005; Taurino et al., 2010). This technology provides the opportunity to explore altered expression of large numbers of genes simultaneously by use of e.g. quantitative polymerase chain reaction ( $q P C R$ ), RNA Sequencing or microarrays; and may lead to a better understanding of the regulatory events involved in health and disease processes. In the field of environmental health, human biomonitoring studies are used to investigate possible associations between lifestyle, environmental factors and public health. Also in this domain, researchers have adopted genome-wide transcriptome analysis of human matrices such as whole blood in order to identify expression signatures reflecting early biological responses (Hebels et al., 2013; Remy et al., 2014; van Leeuwen et al., 2008). Peripheral blood is the most practical tissue to profile gene expression of the human immune system due to accessibility, whereas the site of primary infection in certain diseases may not be easy to access, accurate reflection of in vivo processes at the time of blood collection and the possibility to obtain reproducible blood transcriptional profiles from volumes as small as that obtained from a finger prick (Blankley et al., 2014; Vartanian et al., 2009; Weber et al., 2010). Due to ex vivo instability of RNA transcripts (Baechler et al., 2004; Fleige \& Pfaffl, 2006a; Fleige et al. 2006b; Hartel et al., 2001), a crucial challenge for gene expression analysis on blood samples is rapid sample handling and mRNA stabilization by adding RNA preservative (e.g. PAXgene ${ }^{T M}$ Blood RNA Tube, Tempus $^{T M}$ Blood RNA tube, RNAlater ${ }^{\circledR}$ Stabilization Reagent). The latter stabilizes RNA and prevents induction of new transcripts (Asare et al., 2008; Rainen et al., 2002). Quick addition or direct collection of blood in RNA preservative is a significant challenge during fieldwork in biomonitoring studies and is even not always possible for example in case of cord blood cell collection or prior fractionation of blood cell populations.

Recent studies have investigated gene instability in whole blood collected immediately in a blood tube versus different bench times (hours [hr] or days), collection with or without RNA preservative or anticoagulants, and storage at different temperatures or for extended periods of time. In this context, bench time is considered as the time between blood collection and sample stabilization, either through addition of RNA preservative or by storage at low temperature. Differences in gene expression were mainly observed between different bench times ( $2 \mathrm{hr}$ minimum), followed by anticoagulants and to a much lesser extent storage temperatures and duration (Duale et al., 2012, 2014; Hebels et al., 2013). Yet, no study has evaluated the effects of short bench times (as short as 10 minutes [min] to $30 \mathrm{~min}$ after collection) on whole blood gene expression profiling while this actually is a realistic scenario in sampling protocols of large-scale population studies.

This study focuses on the effect of short bench times (less than $30 \mathrm{~min}$ ) and RNA stabilization buffer on RNA yield, RNA quality and specific gene expression changes in whole blood obtained from three healthy donors, requiring the comparison of two commercially available stabilizing technologies i.e. Tempus $^{\text {TM }}$ Blood RNA tube and RNAlater ${ }^{\circledR}$ Stabilization Reagent. In order to obtain an overall picture of the influence
84

85 
of short bench time on gene expression, whole transcriptome and pathway analysis was performed on RNA derived from whole blood collected directly in a Tempus ${ }^{\mathrm{TM}}$ Blood RNA Tube versus blood that was mixed with RNAlater ${ }^{\circledR}$ Stabilization Reagent 30 min after collection. In a next step, the effect of even shorter bench times (as short as $10 \mathrm{~min}$ ), was evaluated for the most affected molecule using qPCR.

\section{Methods}

\section{Ethical statement}

This study protocol was approved by the ethical committee of the University of Antwerp (B300201316329).

\section{Sample collection and storage}

Whole blood samples were obtained via phlebotomy from three healthy adult female donors aged 33.4, 32.4 and 23.5 years. From each participant peripheral blood was collected in two Tempus ${ }^{\mathrm{TM}}$ Blood RNA tubes and in two dipotassium ethylenediaminetetraacetic acid (EDTA) Vacutainer ${ }^{\mathrm{TM}}$ tubes (Becton Dickinson, Franklin Lakes, NJ). The duplicate Tempus $^{\mathrm{TM}}$ Blood RNA tubes from one donor (donor A) were further used in the experiment as technical replicates. After collection, the EDTA blood sample kept at room temperature was processed in different ways: (1) $3 \mathrm{~mL}$ was added to the Tempus $^{\mathrm{TM}}$ Blood RNA tubes after $10 \mathrm{~min}$ and $30 \mathrm{~min}$; and (2) $0.5 \mathrm{~mL}$ blood was mixed with $1.3 \mathrm{~mL}$ RNAlater ${ }^{\circledR}$ Stabilization Reagent immediately after blood drawing and after $10 \mathrm{~min}$, $20 \mathrm{~min}$ and $30 \mathrm{~min}$ (Table 1). A similar sampling procedure was used for both stabilization buffers in order to compare RNA yield, RNA quality and specific gene expression changes between the two stabilization methods $10 \mathrm{~min}$ or $30 \mathrm{~min}$ after blood collection. To this end, the blood was collected in a plain EDTA tube and after 10 min the Tempus $^{\mathrm{TM}}$ Reagent or the RNAlater ${ }^{\circledR}$ Stabilization Reagent was added. After sample collection, the Tempus ${ }^{\mathrm{TM}}$ Blood RNA tubes were kept at ambient temperature and the blood- RNAlater ${ }^{\circledR}$ Stabilization Reagent aliquots were transferred to dry ice, as indicated by the respective manufacturers. Upon arrival at the laboratory, the Tempus ${ }^{\mathrm{TM}}$ Blood RNA tubes were stored at $-20^{\circ} \mathrm{C}$ and the blood-RNAlater ${ }^{\circledR}$ Stabilization Reagent aliquots were stored at $-80^{\circ} \mathrm{C}$ as recommended by the manufacturers. The duration of cold storage of the stabilized blood fraction prior to transcriptome analysis was one week.

Table 1. Sampling conditions for each individual.

\begin{tabular}{|c|c|c|c|c|}
\hline \multirow[b]{2}{*}{ RNA stabilization buffer } & \multicolumn{4}{|c|}{$\begin{array}{l}\text { Time between collection and addition of RNA } \\
\text { preservative (bench time) }\end{array}$} \\
\hline & 0 min & $10 \mathrm{~min}$ & $20 \mathrm{~min}$ & $30 \mathrm{~min}$ \\
\hline Tempus & $x^{*}$ & $x$ & - & $x$ \\
\hline RNAlater & $x$ & $\mathrm{X}$ & $x$ & $x^{*}$ \\
\hline \multicolumn{5}{|c|}{$\begin{array}{l}\text { Whole blood was directly collected in a Tempus }{ }^{\mathrm{TM}} \text { Blood RNA Tube or mixed } \\
\text { with RNAlater }{ }^{\circledR} \text { Stabilization Reagent. After } 10,20 \text { and/or } 30 \text { min, whole blood } \\
\text { was added to the Tempus }{ }^{\mathrm{TM}} \text { Blood RNA tubes or was mixed with RNAlater }{ }^{\circledR} \\
\text { Stabilization Reagent. All sampling conditions were used for quantitative qPCR; } \\
\text { sampling conditions applied for microarray analysis are indicated with *. }\end{array}$} \\
\hline
\end{tabular}

\section{RNA extraction and QC}

Frozen samples were thawed at room temperature and RNA was isolated immediately using the Tempus ${ }^{\mathrm{TM}}$ Spin RNA Isolation Kit (Ambion, Austin, TX, USA) and the RiboPure Blood Kit (Ambion, Austin, TX, USA) according to the manufacturer's instructions. The concentration of extracted total RNA was measured before and after DNase I treatment (DNAfree $^{\mathrm{TM}} \mathrm{Kit}_{\text {, Invitrogen[AQ2) }}$ using the NanoDrop ND-1000 spectrophotometer (NanoDrop Technologies, Wilmington, DE, USA). RNA purity was estimated by examining the OD 260/ 280 and the OD 260/230 ratios. After DNase I treatment, RNA integrity was assessed by the Agilent 2100 Bioanalyzer using the Agilent RNA 6000 Nano Kit (Agilent Technologies, Palo Alto, CA, USA). The RNA integrity numbers (RIN) were derived using the Agilent 2100 Expert Software $(R I N=1$; low RNA quality to $\mathrm{RIN}=10$; highest RNA quality).

\section{RNA amplification and labelling}

Total RNA was amplified and labelled to generate complementary RNA (cRNA) using the Low Input Quick Amp Labelling (two colour) kit from Agilent Technologies. Briefly, an aliquot of 75-100 ng of RNA was reverse transcribed into complementary DNA (CDNA) using T7-promotor primer and the Affinity Script enzyme. The CDNA was transcribed into CRNA which was fluorescently labelled by simultaneous incorporation of cyanine 3-CTP (Су3) for the Tempus ${ }^{\mathrm{TM}}$ Blood RNA tubes and cyanine 5-CTP (Cy5) for the blood-RNAlater ${ }^{\circledR}$ Stabilization Reagent aliquots. The single-stranded, labelled CRNA was purified with Qiagen's RNeasy mini spin columns (Qiagen, KJ Venlo, Netherlands). cRNA yield and Cy-incorporation efficiency (specific activity) were determined using the NanoDrop spectrophotometer.

\section{Microarray analysis and data pre-processing}

From each donor, equal amounts ( $825 \mathrm{ng}$ ) of Cy3- and Cy5labelled CRNA derived from whole blood collected directly in TempusTM Blood RNA Tubes or mixed with RNAlater ${ }^{\circledR}$ Stabilization Reagent $30 \mathrm{~min}$ after collection were competitively hybridized onto $4 \times 44 \mathrm{~K}$ Agilent Whole Human Genome microarrays (design 014850) for $17 \mathrm{hr}$ using the automated HS4800TM Pro Hybridization Station according to the manufacturer's instructions (Tecan, Männedorf, Switzerland). The arrays were scanned on an Agilent DNA microarray scanner (G2565BA) and further processed using Agilent Feature Extraction Software (Version 10.7) . The software automatically finds and places microarray grids, rejects outlier pixels, accurately determines feature intensities and ratios, and calculates statistical confidences. For each feature on the array, the software accurately determines the intensity for the red (derived from Cy5 fluorescence) and the green signal (derived from Cy3 fluorescence), which were used as input for further processing and analysis (Matlab, version R2012b). Normalization includes both linear scaling and lowest normalization to a rank invariant set of microarray features. Changes in gene expression relative to the control Tempus ${ }^{\mathrm{TM}}$ Blood RNA tube (also defined as 2-base logarithmic fold change [LFC]) were 
obtained by calculating the ratio between rProcessedSignal (RNAlater Stabilization Reagent) and gProcessedSignal (Tempus $^{\mathrm{TM}}$ Blood RNA tube). Fold changes (FC) were transformed by a 2-base logarithm in order to have distributions that are closer to Gaussian ones. Note that a strictly positive or negative LFC means an up- or down-regulation of the corresponding gene expression level. A LFC of 1 and -1 is representative for a fold change of 2 and 0.5 , respectively. The control probes were removed and data files with the expression signals of 41000 unique probes were obtained.

\section{Pathway and network analysis}

Functional and pathway enrichment assessment was conducted using Ingenuity Pathway Analysis (IPA) software (Ingenuity $^{\circledR}$ Systems, Inc., Mountain View, CA, USA). Expression change differences between collection in a tube with RNAlater ${ }^{\circledR}$ Stabilization Reagent after 30 min and direct collection in a Tempus ${ }^{\mathrm{TM}}$ Blood RNA Tube within one donor with a $p$ value $\leq 0.01$ (from the Feature Extraction files) and a LFC $\geq 1$ were considered significant. For the union of significant genes, the median LFC for each transcript was calculated over the three donors. Network relationships between the genes were created based on information contained in Ingenuity's Knowledge Base by entering these values in the IPA core analysis. Three criteria were set before adding relationships and molecules. First, only direct relationships were allowed. Second, only a subset of molecules that was previously described in human peripheral blood cells (blood platelets, hematopoietic progenitor cells and immune cells) was considered for pathway analysis. And third, all types of interaction molecules were accepted in the network. Upstream Regulator Analysis (URA) was used to identify the cascade of upstream transcriptional regulators (transcription factors, enzyme, cytokine, growth factor, miRNA, compound or drug) that could explain the observed gene expression changes in the dataset. For each potential transcriptional regulator an overlap $p$ value was computed with Fisher's Exact test to assign a score to each subnetwork that indicates the likelihood of the input genes in the network being found together due to random chance; and an activation $z$-score as well to infer the activation status of the predicted transcriptional regulators. Significance is generally attributed to $p$ values $<0.01$ and $z$-scores $\leq-2$ or $\geq 2$. Further details of these calculations can be obtained at: http://pages.ingenuity.com/ln genuityUpstreamRegulatorAnalysisWhitepaper.html. Moreover, Canonical Pathway Analysis was used to identify the known pathways according to the Ingenuity Knowledge Base associated with metabolism and signalling that were most significant to the dataset. The significance of the association between the dataset and the canonical pathway was measured in two ways: 1) A ratio of the number of molecules from the dataset that map to the pathway divided by the total number of molecules that map to the canonical pathway was calculated. 2) Fisher's Exact test was used to calculate a $p$ value determining the probability that the association between the genes in the dataset and the canonical pathway is explained by chance alone (Cohen et al., 2014). $p$ Values were adjusted for multiple testing using the Benjamini-Hochberg method (B-H $p$ value) (Benjamini \& Hochberg, 1995).

\section{Quantitative PCR}

295

Total RNA from samples was used as template for the synthesis of cDNA using the Transcriptor First Strand cDNA Synthesis Kit following the instructions of the manufacturer (Roche, Vilvoorde, Belgium). The amplification reactions were carried out in a Veriti 96-well Thermal Cycler (Applied Biosystems). qPCR was performed in 384-well plates using a Roche LightCycler480, The reaction mixtures consisted of $2.5 \mu \mathrm{L}$ cDNA sample and $7.5 \mu \mathrm{L}$ reaction mix $(5 \mu \mathrm{L}$ LightCycler480 Probes Master Mix (2x; Roche), $0.5 \mu \mathrm{L}$ PrimeTime ${ }^{\circledR}$ qPCR Assays (20x; Integrated DNA Technologies, Leuven, Belgium) and $2 \mu \mathrm{L}$ nuclease-free water). Details of the $5^{\prime}$ exonuclease PrimeTime ${ }^{\circledR}$ qPCR Assays that were used are given in Table 2. The qPCR programme was set to one cycle of pre-incubation at $95^{\circ} \mathrm{C}$ for $10 \mathrm{~min}$ followed by 45 cycles of amplification ( 10 seconds [s] at $95^{\circ} \mathrm{C}, 30 \mathrm{~s}$ at $62^{\circ} \mathrm{C}$ and $1 \mathrm{~s}$ at $72^{\circ} \mathrm{C}$ ) and one cycle of cooling at $40^{\circ} \mathrm{C}$ for $10 \mathrm{~s}$. qPCR data processing was performed using the LightCycler software (Bos et al., 2012). Gene expression changes were analysed using the Biogazelle qBasePlus software (www.qbaseplus.com; Biogazelle, Ghent, Belgium) and were calculated as relative FC with the $\Delta \Delta C \mathrm{Ct}$ method (Hellemans et al., 2007). The stability of three reference genes (GNB2L1, RPLPO, RPL13A) was assessed as described by Vandesompele et al. (2002). To achieve accurate $\mathrm{FC}$, normalization was performed to a set of at least three stable reference genes. Subsequently, FCs were transformed by a 2-base logarithm and addressed as LFC.

\section{Statistical data analysis}

Statistical analyses were performed using SPSS (version 22.0; IBM Corp, Armonk, NY, USA) and Matlab R2012b (MathWorks, Natick, MA, USA). The values of RNA yield and RNA integrity were visually inspected and presented in a table as the mean\pm standard deviation (SD) for all conditions. Statistical comparisons were made using a two-way repeated measures analysis of variance (ANOVA) with time and tube serving as within-subjects factors and the data (RNA yield and RIN values) logarithmic transformed to be a better fit to the normal distribution. Regarding the qPCR data, statistical differences between the different stabilization systems were evaluated using a paired $t$-test. Significant between-group differences were tested by using an independent sample $t$-test. The level of significance of all statistical analyses was set at $p=0.05$.

\section{Results}

The effect of short bench times on RNA yield and quality

To evaluate the effect of short bench times on the stability of total RNA in whole blood, obtained from three healthy donors, RNA yield and quality of all RNA samples were determined. Bench time was defined as the time between blood
296

297

298

299

300

301

302 
Table 2. Sequences of the $5^{\prime}$ exonuclease PrimeTime ${ }^{\circledR}$ assays.

407

IL-8: NM_000584 (reference gene)

PrimeTime Probe

PrimeTime Primer 2 (reverse)

PrimeTime Primer 1 (forward)

GNB2L1: NM_006098 (reference gene)

PrimeTime Probe

PrimeTime Primer 2

PrimeTime Primer 1

RPLPO: NM_001002 (reference gene)

PrimeTime Probe

PrimeTime Primer 2

PrimeTime Primer 1

RPL13A: NM_012423 (reference gene)

PrimeTime Probe

PrimeTime Primer 2

PrimeTime Primer 1
/56-FAM/AGGACAAGA/ZEN/GCCAGGAAGAAACCAC/3IABkFQ/

ACACAGAGCTGCAGAAATCAG

TITCAGAGACAGCAGAGCAC

/56-FAM/AGTGGCTCT/ZEN/CATCCTGGACAGTGTAT/3IABkFO/

CAAGCTATGGAATACCCTGGG

AGAAGCGGACACAAGACAC

/56-FAM/CCCTGTCTT/ZEN/CCCTGGGCATCAC/3IABkFQ/

TCGTCTITAAACCCTGCGTG

TGTCTGCTCCCACAATGAAAC

/56-FAM/CTTCAGACG/ZEN/CACGACCTTGAGGG/3IABkFQ/ CTGTCACTGCCTGGTACTTC TGTTTGACGGCATCCCAC
408

409

410

411

412

413

414

415

416

417

418

419

420

421

422

423

424

425

426

427

428

429

430

431

432

433

434

435

436

437

438

439

440

441

442

443

444

445

446

447

448

449

450

451

452

453

454

455

456

457

458

459

460

461

462

463

464 transcriptome analysis was performed on RNA derived from whole blood collected directly in a Tempus ${ }^{\mathrm{TM}}$ Blood RNA Tube versus blood that was mixed with RNAlater ${ }^{\circledR}$

Using the gene set that was altered in expression by the $30^{\prime}$ bench time, enriched pathways and biological processes were identified. The transcriptional regulator that can explain the observed gene expression changes in the three donors was the Nuclear factor of kappa light polypeptide gene enhancer in B-cells inhibitor, alpha (NFKBIA) with an overlap $p$ value of 

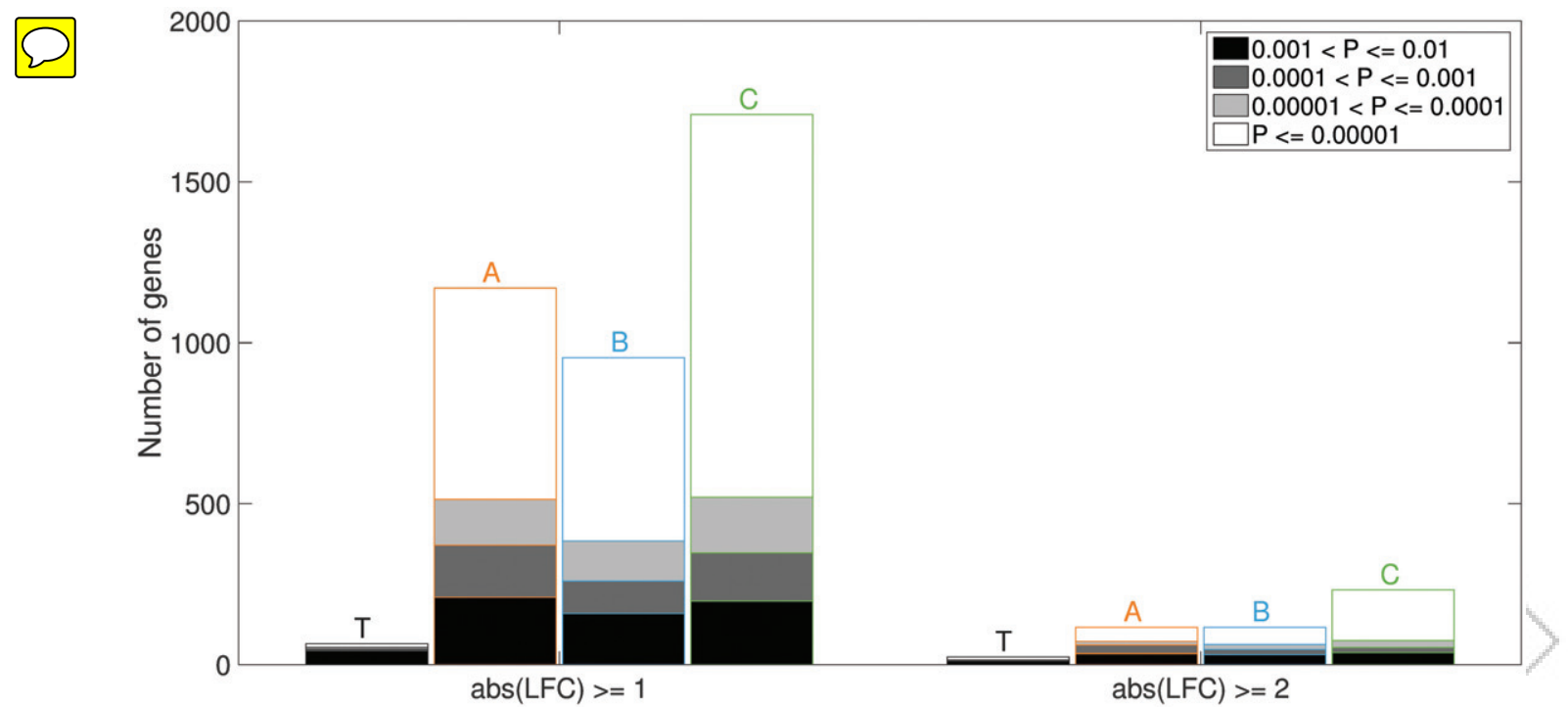

Figure 1. Differences in gene expression between the two stabilization systems. Number of genes significantly different between collection in a tube with RNAlater ${ }^{\circledR}$ Stabilization Reagent at $30 \mathrm{~min}$ and in a Tempus ${ }^{\top \mathrm{M}}$ Blood RNA Tube at 0 min for the three donors (A, B, C) with a $p$ value $\leq 0.01$ and an abs(LFC) $\geq 1$ (left) or 2 (right). The technical control $\mathrm{T}$ includes genes that differ between two Tempus ${ }^{\mathrm{TM}}$ Blood RNA Tubes derived from individual $\mathrm{A}$ at $0 \mathrm{~min}$.
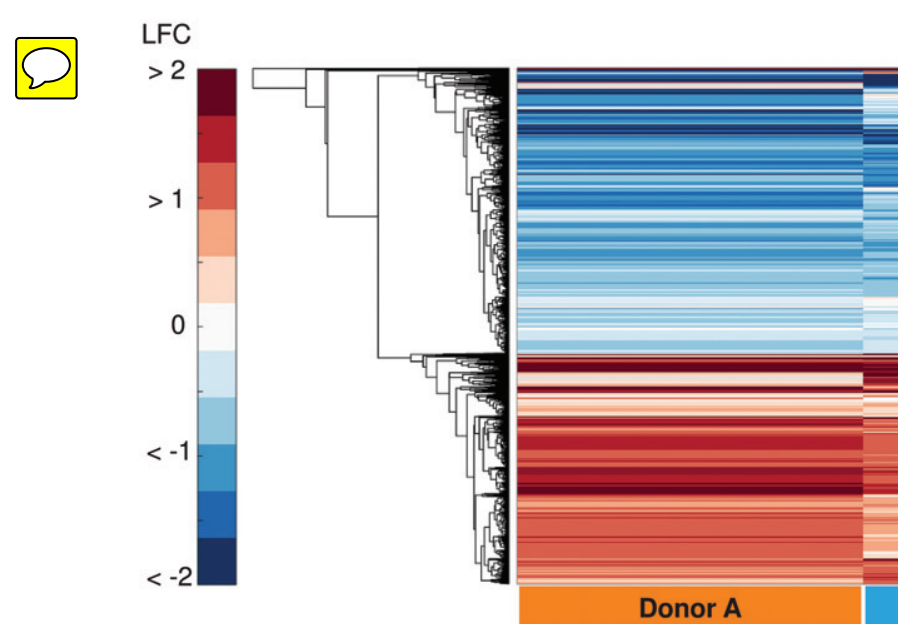

Figure 2. Expression change of the significantly different genes $(n=2356)$ between collection in a tube with RNAlater ${ }^{\circledR}$ Stabilization Reagent after 30 min and direct collection in a Tempus ${ }^{\mathrm{TM}}$ Blood RNA Tube. The gene expression changes are indicated as LFC by a gradient of blue and red colour, as shown in the color scale on the left side of the heat map. The red colour indicates upregulated gene expression, the blue colour indicates downregulated gene expression. Additionally, the genes were clustered based on their LFC (y-axis).

7.99E - 07 and an activation z-score of 2.65. An activation z-score greater than 2 is considered highly statistically significant. NFKBIA was associated with 79 differentially expressed genes in our dataset, and the expression profiles of these genes reflected NFKBIA activation. The NFKBIA-driven mechanistic network, involving 357 genes of our dataset, consisted of 12 transcription regulators. The molecules are coloured in Figure 3 by their predicted activation state: activated (orange) or inhibited (blue). The canonical pathways most significantly associated with the 2356 differentially expressed genes are presented in Table 4. Additionally, IPA calculated a ratio of genes differentially expressed within each canonical pathway and also included in the dataset. The two pathways most significantly associated with the differentially expressed genes are the Molecular Mechanisms of Cancer pathway (B-H $p$ value $=1.2 \mathrm{E}-03 ;$ ratio $=73 / 328)$ and the Glucocorticoid Receptor Signalling pathway $(\mathrm{B}-\mathrm{H} \quad p$ value $=2.6 \mathrm{E}-03$; ratio $=59 / 259)$.
The effect of shorter bench times on interleukin-8 gene activity

The gene, with the maximum abs(LFC) in the microarray experiment, is the pro-inflammatory marker gene interleukin8 (IL-8) with a LFC of 5.083 ,To further scrutinize the effect of short bench times before RNA stabilization, the activity of $I L-8$ in whole blood that was collected in a Tempus ${ }^{\mathrm{TM}}$ Blood RNA Tube or in a tube with RNAlater ${ }^{\circledR}$ Stabilization Reagent after several minutes (0-30 min) was evaluated using qPCR. Figure 4 presents the mean expression change of IL-8 (LFC) for all three donors in whole blood collected in a Tempus ${ }^{\mathrm{TM}}$ Blood RNA Tube (grey bars) or in a tube with RNAlater ${ }^{\circledR}$ Stabilizing Reagent (white bars) after different bench times. The mean expression values are considered relative to the Tempus $^{T M}$ Blood RNA Tube at $0 \mathrm{~min}$. The response of the three donors is shown in orange (A), blue (B) and green (C). Significant differences in expression values of $I L-8$ between 

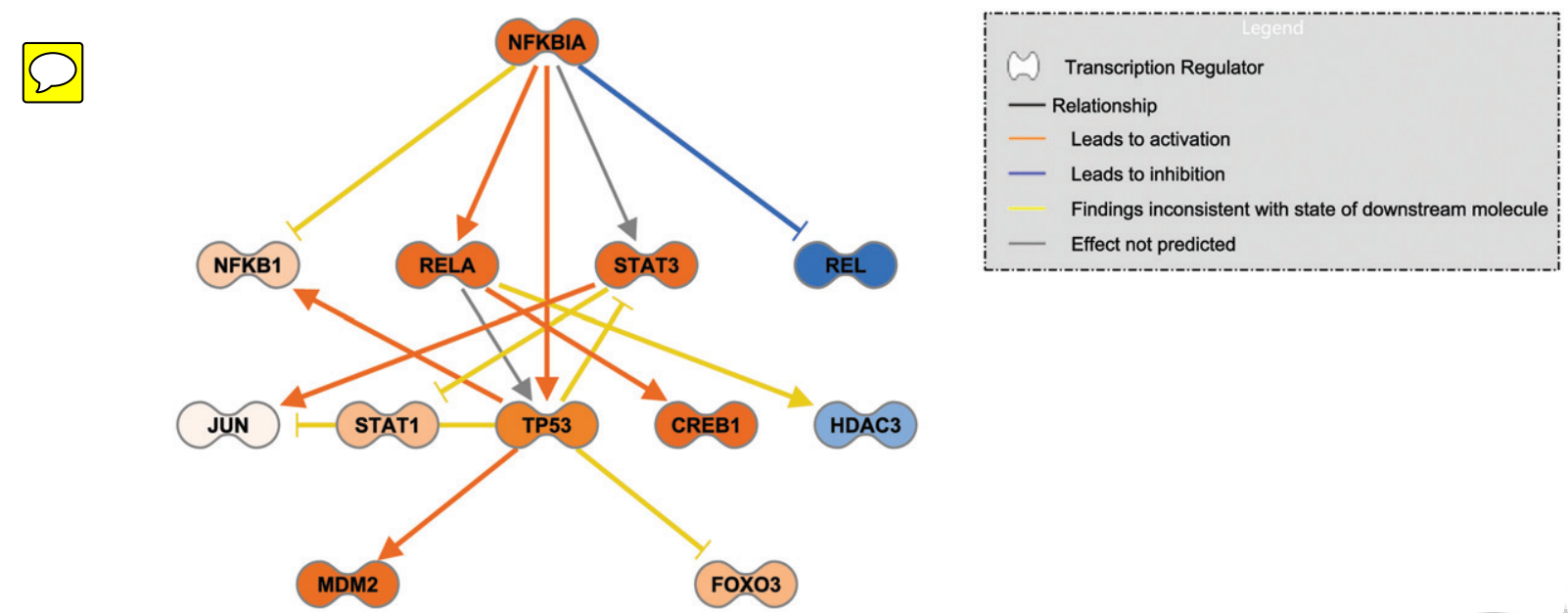

Fox03

Figure 3. NFkBIA-driven mechanistic network. This network, involving 357 genes of the 2356 dataset genes, consists of 12 transcription regulators. The lines connecting the nodes are coloured orange when leading to activation of the downstream node, blue when leading to its inhibition, and yellow if the findings underlying the relationship are inconsistent with the state of the downstream node. Pointed arrowheads indicate that the downstream node is expected to be activated if the upstream node connected to it is activated, while blunt arrowheads indicate that the downstream node is expected to be inhibited if the upstream node that connects to it is activated.

Table 4. Top ingenuity canonical pathways related to the 2356 differentially expressed genes.

\begin{tabular}{lcr}
\hline Canonical pathway & B-H $p$ value* & \multicolumn{1}{c}{ Ratio } \\
\hline Molecular mechanisms of cancer & $1.2 \mathrm{E}-03$ & $73 / 328(22.3 \%)$ \\
Glucocorticoid receptor signalling & $2.6 \mathrm{E}-03$ & $59 / 259(22.8 \%)$ \\
Role of NFAT in cardiac hypertrophy & $4.09 \mathrm{E}-02$ & $37 / 158(23.4 \%)$ \\
Role of tissue factor in cancer & $4.44 \mathrm{E}-02$ & $26 / 100(26.0 \%)$ \\
IL-22 signalling & $4.44 \mathrm{E}-02$ & $10 / 24(41.7 \%)$ \\
RAR activation & $4.44 \mathrm{E}-02$ & $37 / 165(22.4 \%)$ \\
Aryl hydrocarbon receptor signalling & $4.44 \mathrm{E}-02$ & $29 / 120(24.2 \%)$ \\
Role of JAK family kinases in IL-6-type & $4.44 \mathrm{E}-02$ & $10 / 25(40.0 \%)$ \\
$\quad$ cytokine signalling & & \\
p53 signalling & $4.57 \mathrm{E}-02$ & $23 / 90(25.6 \%)$ \\
HMGB1 signalling & $4.57 \mathrm{E}-02$ & $27 / 112(24.1 \%)$ \\
\hline
\end{tabular}

The significance of the association between the dataset and the canonical pathway was measured by a $p$ value determining the probability that the association is explained by chance alone, and a ratio of the number of molecules from the dataset that map to the pathway divided by the total number of molecules that map to the canonical pathway.

*Benjamini-Hochberg corrected $p$ value.

the two stabilizing systems $(p=0.004)$ and over different bench times $(p=0.028)$ were observed. Stabilization with RNAlater $^{\circledR}$ Stabilizing Reagent compared to stabilization in a Tempus $^{T M}$ Blood RNA Tube resulted in a $\sim 2$-fold higher induction of $I L-8$. The difference in mean expression value between the two stabilizing systems and expressed in LFC was largest at $0 \mathrm{~min}$ i.e. $1.12(p=0.0083)$. When leaving the blood sample on the bench at room temperature for $10 \mathrm{~min}$ and $30 \mathrm{~min}$, the mean $I L-8$ expression was increased with respectively $28.3 \%(p=0.0300)$ and $15.4 \%(p=0.0259)$ in RNAlater $^{\circledR}$-stabilized samples compared to Tempus $^{\mathrm{TM}}$ stabilized samples. Since no Tempus ${ }^{\mathrm{TM}}$ Blood RNA solution was added to whole blood after 20 min, no comparison with RNAlater ${ }^{\circledR}$ Stabilizing Reagent could be made for this condition. Noteworthy, there was no statistical difference in absolute gene expression values of $I L-8$ between the $\mathrm{qPCR}$ and the above-mentioned microarray experiment $(p=0.762)$.

\section{Discussion}

Transcriptome analysis of peripheral blood as surrogate tissue has become an important approach in clinical and health

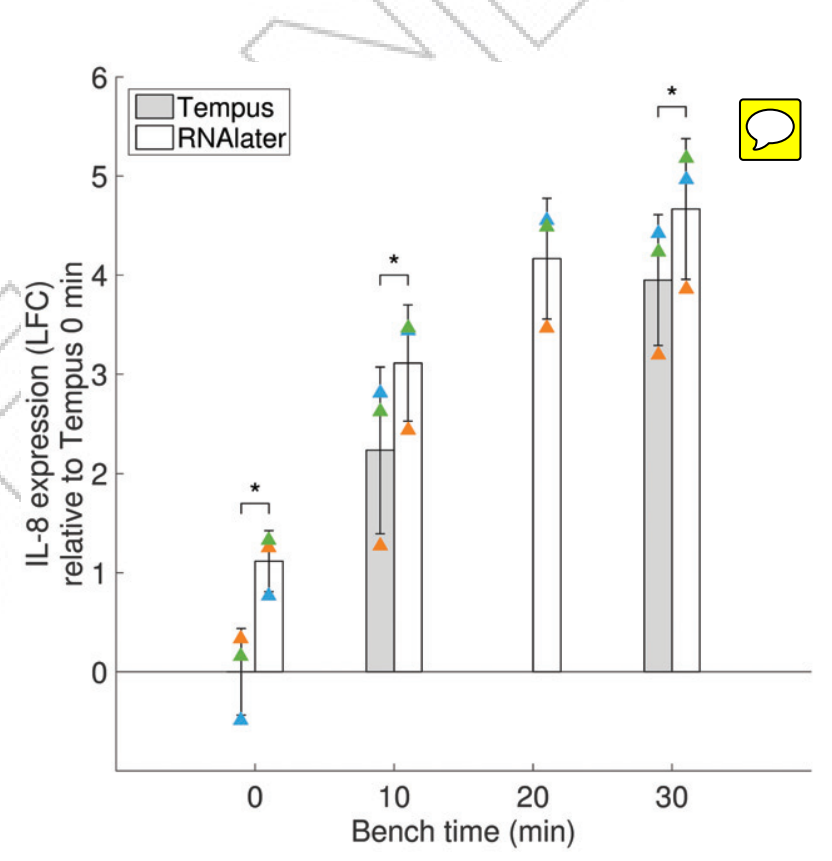

Figure 4. Comparison of IL-8 expression in Tempus ${ }^{\mathrm{TM}}$ and RNAlater ${ }^{\circledR}$ stabilization buffer after different bench times. The mean $I L-8$ expression was examined after the addition of whole blood in a Tempus ${ }^{\mathrm{TM}}$ Blood RNA Tube (grey) or in a tube with RNAlater ${ }^{\circledR}$ Stabilization Reagent (white) after several minutes (0-30 min; $x$-axis). The response of donor $A$ (orange), B (blue) and C (green), The $y$-axis shows the LFC, the difference in expression compared to the Tempus ${ }^{T M}$ Blood RNA Tube at $0 \mathrm{~min}$. Significant differences between collection of whole blood in a Tempus ${ }^{\mathrm{TM}}$ Blood RNA Tube or mixed in a tube with RNAlater ${ }^{\circledR}$ Stabilization Reagent within an individual are indicated with ${ }^{*}(p<0.05)$. The error bars on the graph represent the mean standard deviation of the three donors for each collection condition.

monitoring studies. However, application of gene expression profiling in routine settings requires careful consideration of the influence of sample handling and mRNA stabilization on gene expression profile outcome. These results are often biased by decreased sensitivity and reliability due to ex vivo instability of RNA transcripts and no possibility of immediate mRNA fixation. To this end, we evaluated the influence of short bench times (less than $30 \mathrm{~min}$ ) on RNA yield, RNA quality and specific gene expression levels in whole blood samples, while comparing two commercially available stabilizing 
technologies, i.e. Tempus ${ }^{\mathrm{TM}}$ Blood RNA tube and RNAlater ${ }^{\circledR}$ Stabilization Reagent. The key findings can be summarized as follows:

\section{RNA yield and quality}

In both stabilization systems, no differences in RNA quality and integrity were observed over different short bench times. This was consistent with the recent findings of Hebels et al. that focused on the impact of larger bench times (0-24 hr) on gene expression profiles (Hebels et al. 2013). All samples had RIN values of sufficient quality for transcriptome analysis. A significant difference in RNA yield can most probably be explained by differences in initial blood volume: $3 \mathrm{~mL}$ whole blood for the Tempus ${ }^{\mathrm{TM}}$ Blood RNA tube versus $0.5 \mathrm{~mL}$ for the blood-RNAlater ${ }^{\circledR}$ Stabilization Reagent aliquots.

\section{Gene expression}

In order to obtain an overall picture of the influence of short bench time on gene expression, whole transcriptome analysis was performed on blood collected directly in a RNA stabilizing Tempus tube compared with transfer of the collected blood in RNAlater ${ }^{\circledR}$ Stabilization Reagent after 30 min of incubation. Microarray analyses showed significant changes in expression of a considerate part of the transcriptome (2356 genes), with almost all genes the same expression regulation in the three donors. In this gene set, enriched pathways and biological processes were identified with a prominent role for Nuclear factor of kappa light polypeptide gene enhancer in B-cells (NFкB)-, cancer- and glucocorticoid-mediated networks. Similar findings were published by Hebels et al. when looking at larger bench times. The study revealed pathways mainly involved in apoptosis, stress signalling and DNA damage repair (Hebels et al. 2013). In the current study, network analysis reveals NFKBIA as the most significant and activated upstream regulator of the genes whose expressions were altered by the $30^{\prime}$ bench time. Induction of the NFKBIA protein results in inhibition of $N F K B$, which are key regulators in immune-mediated inflammatory processes, by trapping the activated NFKB in inactive cytoplasmic complexes (Auphan et al., 1995; Ghosh et al., 1998; Hoesel \& Schmid, 2013; Jacobs \& Harrison, 1998; Mogensen, 2009). The two pathways most significantly associated with the differentially expressed genes are the Molecular Mechanisms of Cancer pathway and the Glucocorticoid Receptor Signalling pathway. Glucocorticoids are among the most potent anti-inflammatory and immunosuppressive agents. They inhibit synthesis of almost all known cytokines and of several cell surface molecules required for immune function; and are known to be dominant inhibitors of NFKB activation (Auphan et al., 1995). Besides, inhibition of $\mathrm{NFKB}$ is an important molecular pathway in the protection against cancers (Lin et al., 2010).

The gene mainly altered in the microarray experiment and also involved in NFKBIA- and glucocorticoid-mediated networks, is the pro-inflammatory marker gene $I L-8$. In a next step, the effect of bench times even shorter than 30 minutes was evaluated for this molecule. IL-8 expression levels were demonstrated to be increased over short transcending bench times (0-30 min) in whole blood collected in both stabilization systems. Previous studies demonstrated that IL-8 was induced within $4 \mathrm{hr}$ ex vivo (Barnes et al., 2010; Rainen et al., 2002). Baechler et al. confirmed this dramatic upregulation of IL-8 in overnight samples (Baechler et al. 2004). In our study, at each time point, a $\sim 2$-fold higher induction of $I L-8$ was observed in RNAlater ${ }^{\circledR}$ Stabilizing Reagent compared to the Tempus $^{\text {TM }}$ system. It has been shown that mRNA concentrations after $24 \mathrm{hr}$ storage in PAXgene ${ }^{\mathrm{TM}}$ or Tempus ${ }^{\mathrm{TM}}$ blood collection tubes much more closely approximated the initial values than the grossly induced concentrations in EDTA tubes, indicating that the choice of RNA collection tube greatly influences the transcriptional profiles obtained from peripheral blood (Rainen et al., 2002).

Our data indicated that a large amount of genes are dysregulated by ex vivo incubations of blood as short as $10 \mathrm{~min}$ (as demonstrated for $I L-8)$. The specific cause of the identified gene expression changes should still be determined although several explanations can be hypothesized. Within $30 \mathrm{~min}$ after phlebotomy, the environment of the blood sample can change significantly by e.g. glucose depletion, $\mathrm{pH}$ drop, lactate accumulation, hypoxia and temperature change, causing biological stresses (Barnes et al., 2010; Burczynski \& Rockett, 2006). Even anticoagulant in the collection tube may play a role in modulating cell activation, triggering the release of cytokines (ElHabbal et al., 1995; Pahl \& Brune, 2002). The findings in the present study thus indicate that blood cells that are not immediately stabilized at the moment of phlebotomy, start to compensate for this potentially lethal microenvironment. Their complex regulatory machineries (like e.g. the Glucocorticoid Receptor Signalling pathway) may be activated to regulate the expression of many stress-associated genes, genes associated with important physiological functions like cell cycle, proliferation, transcription and metabolism and genes associated with immune function (e.g. chemokines, cytokine receptors and cell surface receptors). These biological processes and functions can therefore only be interpreted when measured in studies with an appropriate experimental design.

\section{Conclusion}

Overall these findings demonstrate that even short bench times (as short as $10 \mathrm{~min}$ ) affect the human blood transcriptome. The optimal condition for gene expression profiling, in the context of monitoring health and disease processes in large-scale population studies, is direct collection of RNA in a stabilizing system (e.g. Tempus ${ }^{\mathrm{TM}}$ Blood RNA Tube). When direct collection in a RNA stabilization system isn't possible due to practical reasons e.g. volume requirements, RNAlater ${ }^{\circledR}$ Stabilization Reagent can be used instead. For the latter, researchers must take into account that certain genes e.g. stress-associated genes can have higher gene expression levels due to technical variation instead of biological factors, and therefore, caution should be taken when interpreting expression profiles. Although the results based on three individuals are very clear, a larger sample size could add power to fully 
comprehend the impact of short bench time on the human blood transcriptome.

\section{Disclosure statement}

The authors report no declarations of interest.

\section{Funding information}

This work was supported by a PhD fellowship from the University of Antwerp granted towards Carmen Franken and financed by the Ministry of the Flemish Community (Department of Economics, Science and Innovation; Flemish Agency for Care and Health; and Department of Environment, Nature and Energy).

\section{References}

Asare AL, Kolchinsky SA, Gao Z, et al. (2008). Differential gene expression profiles are dependent upon method of peripheral blood collection and RNA isolation. BMC Genomics 9:474.

Auphan N, DiDonato JA, Rosette C, et al. (1995). Immunosuppression by glucocorticoids: inhibition of NF-kappa B activity through induction of I kappa B synthesis. Science 270:286-90.

Baechler EC, Batliwalla FM, Karypis G, et al. (2004). Expression levels for many genes in human peripheral blood cells are highly sensitive to ex vivo incubation. Genes Immun 5:347-53.

Bai JP, Alekseyenko AV, Statnikov A, et al. (2013). Strategic applications of gene expression: from drug discovery/development to bedside. AAPS J 15:427-37.

Barnes MG, Grom AA, Griffin TA, et al. (2010). Gene expression profiles from peripheral blood mononuclear cells are sensitive to short processing delays. Biopreserv Biobank 8:153-62.

Benjamini Y, Hochberg Y. (1995). Controlling the false discovery rate - a practical and powerful approach to multiple testing. J R Stat Soc Ser B Methodol 57:289-300.

Blankley S, Berry MP, Graham CM, et al. (2014). The application of transcriptional blood signatures to enhance our understanding of the host response to infection: the example of tuberculosis. Philos Trans $R$ Soc Lond B Biol Sci 369:20130427.

Bos I, De BP, Int PL, et al. (2012). Negative effects of ultrafine particle exposure during forced exercise on the expression of Brain-Derived Neurotrophic Factor in the hippocampus of rats. Neuroscience 223:131-9.

Burczynski ME, Rockett JC. (2006), Surrogate tissue analysis: genomic, proteomic, and metabolomic approaches. Boca Raton, FL: Taylor \& Francis Group, LLC.

Cohen S, Mosig R, Moshier E, et al. (2014). Interferon regulatory factor 1 is an independent predictor of platinum resistance and survival in highgrade serous ovarian carcinoma. Gynecol Oncol 134:591-8.

Duale N, Brunborg G, Ronningen KS, et al. (2012). Human blood RNA stabilization in samples collected and transported for a large biobank. BMC Res Notes 5:510.

Duale N, Lipkin WI, Briese T, et al. (2014). Long-term storage of blood RNA collected in RNA stabilizing Tempus tubes in a large biobankevaluation of RNA quality and stability. BMC Res Notes 7:633.

ElHabbal MH, Smith L, Elliott MJ, Strobel S. (1995). Effect of heparin anticoagulation on neutrophil adhesion molecules and release of IL8: C3 is not essential. Cardiovasc Res 30:676-81.
Ellinger-Ziegelbauer H, Aubrecht J, Kleinjans JC, Ahr HJ. (2009). Application of toxicogenomics to study mechanisms of genotoxicity and carcinogenicity. Toxicol Lett 186:36-44.

Fleige S, Pfaffl MW. (2006a). RNA integrity and the effect on the real-time qRT-PCR performance. Mol Aspects Med 27:126-39.

Fleige S, Walf V, Huch S, et al. (2006b). Comparison of relative mRNA quantification models and the impact of RNA integrity in quantitative real-time RT-PCR. Biotechnol Lett 28:1601-13.

Ghosh S, May MJ, Kopp EB. (1998). NF-kappa B and Rel proteins: evolutionarily conserved mediators of immune responses. Annu Rev Immunol 16:225-60.

Hartel C, Bein G, Muller-Steinhardt M, Kluter H. (2001). Ex vivo induction of cytokine mRNA expression in human blood samples. J Immunol Methods 249:63-71.

Hebels DG, Georgiadis P, Keun HC, et al. (2013). Performance in omics analyses of blood samples in long-term storage: opportunities for the exploitation of existing biobanks in environmental health research. Environ Health Perspect 121:480-7.

Hellemans J, Mortier G, De PA, et al. (2007). qBase relative quantification framework and software for management and automated analysis of real-time quantitative PCR data. Genome Biol 8:R19.

Hoesel B, Schmid JA. (2013). The complexity of NF-KB signaling in inflammation and cancer. Mol Cancer 12:86.

Jacobs MD, Harrison SC. (1998). Structure of an IkappaBalpha/NF-kappaB complex. Cell 95:749-58.

Lin Y, Bai L, Chen W, Xu S. (2010). The NF-kappaB activation pathways, emerging molecular targets for cancer prevention and therapy. Expert Opin Ther Targets 14:45-55.

McHale CM, Zhang L, Hubbard AE, Smith MT. (2010). Toxicogenomic profiling of chemically exposed humans in risk assessment. Mutat Res 705:172-83.

Mogensen TH. (2009). Pathogen recognition and inflammatory signaling in innate immune defenses. Clin Microbiol Rev 22:240-73.

Opitz L, Salinas-Riester G, Grade M, et al. (2010). Impact of RNA degradation on gene expression profiling. BMC Med Genomics 3:36.

Pahl A, Brune K. (2002). Stabilization of gene expression profiles in blood after phlebotomy. Clin Chem 48:2251-3.

Rainen L, Oelmueller U, Jurgensen S, et al. (2002). Stabilization of mRNA expression in whole blood samples. Clin Chem 48:1883-90.

Remy S, Govarts E, Bruckers L, et al. (2014). Expression of the sFLT1 gene in cord blood cells is associated to maternal arsenic exposure and decreased birth weight. PLoS One 9:e92677

Sullivan PF, Fan C, Perou CM. (2006). Evaluating the comparability of gene expression in blood and brain. Am J Med Genet B Neuropsychiatr Genet 141B:261-8.

Tang Y, Gilbert DL, Glauser TA, et al. (2005). Blood gene expression profiling of neurologic diseases: a pilot microarray study. Arch Neurol 62:210-15.

Taurino C, Miller WH, McBride MW, et al. (2010). Gene expression profiling in whole blood of patients with coronary artery disease. Clin Sci (Lond) 119:335-43.

van Leeuwen DM, Gottschalk RW, Schoeters G, et al. (2008). Transcriptome analysis in peripheral blood of humans exposed to environmental carcinogens: a promising new biomarker in environmental health studies. Environ Health Perspect 116:1519-25.

Vandesompele J, De PK, Pattyn F, et al. (2002). Accurate normalization of real-time quantitative RT-PCR data by geometric averaging of multiple internal control genes. Genome Biol 3:RESEARCH0034.

Vartanian K, Slottke R, Johnstone T, et al. (2009). Gene expression profiling of whole blood: comparison of target preparation methods for accurate and reproducible microarray analysis. BMC Genomics 10:2.

Weber DG, Casjens S, Rozynek P, et al. (2010). Assessment of mRNA and microRNA stabilization in peripheral human blood for multicenter studies and biobanks. Biomark Insights 5:95-102. 